\section{Avaliação de anti-inflamatórios não esteroidais no tratamento da dor de ovinos submetidos à implantação de cânula ruminal e orquiectomia}

\author{
[Assessment of nonsteroidal anti-inflammatory drugs in treatment of pain in sheep submitted to \\ ruminal cannulation and orchiectomy] \\ R.S. Sousa ${ }^{1}$, I.K.F. Sousa ${ }^{2 *}$, L.F. Reis ${ }^{1}$, F.A.L.M. Rodrigues ${ }^{l}$, A.H.H. Minervino ${ }^{3}$, C.S. Mori ${ }^{1}$, \\ M.A.P. Moreira ${ }^{4}$,V.V. Paula ${ }^{5}$, R.A. Barreto Júnior ${ }^{5}$, E.L. Ortolani ${ }^{1}$ \\ ${ }^{1}$ Universidade de São Paulo - São Paulo, SP \\ ${ }^{2}$ Instituto Federal do Amazonas -Manaus, AM \\ ${ }^{3}$ Universidade Federal do Oeste do Pará - Santarém, PA \\ ${ }^{4}$ Instituto Federal Goiano - Urutaí, GO \\ ${ }^{5}$ Universidade Federal Rural do Semiárido - Mossoró, RN
}

R.S. Sousa:

http://orcid.org/0000-0001-9121-1038

I.K.F. Sousa:
http://orcid.org/0000-0002-3337-6368

http://orcid.org/0000-0003-4568-2220 Rodrigue: http://orcid.org/0000-0001-5902-3264 A.H.H. Minervino: http://orcid.org/0000-0002-6742-3652 C.S. Mori:

http://orcid.org/0000-0002-4897-7764 M.A.P. Moreira:

http://orcid.org/0000-0003-2262-5071 R.A. Barreto Júnior:

http://orcid.org/0000-0002-9416-6129 E.L. Ortolani:

http://orcid.org/0000-0002-2439-5364

\begin{abstract}
RESUMO
O presente trabalho objetivou comparar o efeito do flunixin meglumine, cetoprofeno e meloxicam no tratamento da dor pós-operatória de ovinos submetidos à implantação de cânula ruminal e orquiectomia. Foram utilizados 32 ovinos, machos, pesando em média $35,5 \pm 3,5 \mathrm{~kg}$, distribuídos em três grupos: GFlu (flunixin meglumine 1,1 mg/kg i.v.), GCet (cetoprofeno 3,0mg/kg i.v.) e GMel (meloxicam 0,5mg/kg i.v.). Exame clínico e coletas de sangue foram realizados no M0 (pré-avaliação), M1 (10 minutos após a pré-avaliação), M2 (início da sutura para fixação da cânula ruminal), M3 (logo após o término da cirurgia) e em duas, 12, 23, 25, 48 e 72 horas após a cirurgia (M2h, M12h, M23h, M25h, M48h e M72h), quando foram avaliados cortisol, glicose, proteína total, albumina, $\gamma$-glutamiltransferase (GGT), aspartato aminotransferase (AST), creatina quinase (CK), ureia, creatinina e hemograma. Nos M2h, M12h, M23h, M25h e M48h, foi realizada avaliação comportamental. O GFlu apresentou maior concentração de cortisol no M12h e no M48h e maior escore de dor na fístula e no testículo no M12h, quando comparado ao GMel. Os animais do GCet apresentaram menor interação com outros membros da baia no M23h. A ação analgésica do meloxicam foi maior em animais submetidos à implantação de cânula ruminal e orquiectomia, quando comparado ao flunixin meglumine e ao cetoprofeno.
\end{abstract}

Palavras-chave: estresse, bem-estar, comportamento, analgésico, cicloxigenase

\begin{abstract}
This study aimed to compare the effect of flunixin meglumine, ketoprofen, and meloxicam in the treatment of postoperative pain in sheep submitted to ruminal cannulation and orchiectomy. 32 sheep were submitted to implantation of rumen cannula and orchiectomy, divided into three groups: GFlu (Flunixin meglumine 1,1mg/kg i.v.); GCet (Ketoprofen 3,0mg/kg i.v.); GMel (Meloxicam 0,5mg/kg i.v.). Clinical examination and blood samples were performed at MO (pre-evaluation), M1 (10 minutes after pre-evaluation), M2 (beginning ruminal cannula), M3 (immediately post-surgery), and M2h, M12h, M23h, M25h, M48h and M72h $(2 h, 12 h$, $23 \mathrm{~h}, 25 \mathrm{~h}, 48 \mathrm{~h}$ and 72 hours post-surgery) with the evaluation of cortisol, glucose, total protein, albumin, $\gamma-$ glutamyl transferase (GGT), aspartate aminotransferase (AST), creatine kinase (CK), urea, creatinine and blood count. At M2h, M12h, M23h, M25h and M48h a behavioral evaluation was performed. The GFlu showed higher concentration of cortisol at $M 12 \mathrm{~h}$ and M48h and greater pain score related with fistula and testis procedures at M12h when compared to GMel. Animals in the GCet group presented lower interaction with other animals in the same M23h paddock. The analgesia provided by Meloxicam was higher than flunixin meglumine and ketoprofen in animals submitted to placement of ruminal cannula and orchiectomy.
\end{abstract}

Keywords: stress, welfare, behavior, analgesic, cyclooxygenase

Recebido em 19 de abril de 2017

Aceito em 7 de dezembro de 2018

*Autor para correspondência (corresponding author)

E-mail: isadora.sousa@ifam.edu.br 


\section{INTRODUÇÃO}

Os ruminantes são considerados animais estoicos, e essa característica talvez esteja ligada à sua história evolutiva. Por serem alvos fáceis de predadores, eles são capazes de suportar diferentes graus de desconforto ou dor sem demonstrar fragilidade ou evidenciarem sinais (Fitzpatrick et al., 2006; Silva et al., 2011). Essa característica, possivelmente, retardou o desenvolvimento de métodos de reconhecimento e de avaliação da dor, ou mesmo favoreceu a pouca utilização de fármacos analgésicos ao longo dos anos, após lesões ou outros eventos nocivos (Molony e Kent, 1997).

Atualmente, embora esteja claro que os ruminantes são sensíveis à dor e que o controle da dor pós-cirurgia melhora a recuperação desses animais (Muir e Woolf, 2001), recente estudo brasileiro demonstrou que apenas $74,4 \%$ dos veterinários de bovinos utilizam analgésicos após procedimentos cirúrgicos, contra $95,3 \%$ dos veterinários de equinos (Lorena et al., 2013); o mesmo acontece em países do Reino Unido, onde apenas $50 \%$ dos veterinários indicam terapia analgésica após cesariana, 55\% após a amputação de dígito e apenas $1 \%$ após descorna (Whay e Huxley, 2005).

Nesse contexto, os fármacos mais utilizados para o controle da dor na clínica de ruminantes são os anti-inflamatórios não esteroidais (AINEs), devido ao baixo preço e aos poucos efeitos colaterais (Lorena et al., 2013). Os AINEs são medicamentos conhecidos pelos seus efeitos inibitórios sobre as cicloxigenases 1 e 2 (COX-1 e COX-2), impedindo a formação de prostaglandinas, que reduz o processo inflamatório. A COX-1 é uma enzima constitutiva importante para o sistema gastrointestinal, os rins e as plaquetas, enquanto a COX-2 é uma enzima indutiva, cuja expressão é aumentada devido a citocinas, nos sítios de inflamação (Adegboyega e Olodade, 2004; Lees et al., 2004; Whay et al., 2005). Dessa forma, AINEs que possuem maior ação sobre a COX-1 podem provocar efeitos deletérios sobre os sistemas em que ela é constituinte.

Entre os AINEs, o flunixin meglumine é considerado um fármaco seletivo para $\mathrm{COX}-1$, o cetoprofeno possui ação mediana sobre $\mathrm{COX}-1$ e maior sobre COX-2, enquanto o meloxicam é considerado um AINE majoritariamente seletivo para COX-2, promovendo menores efeitos colaterais (Whay et al., 2005; Coetzee et al., 2009).

A orquiectomia é a cirurgia mais comum em pequenos ruminantes, sendo utilizada para facilitar o manejo, aprimorar a qualidade da carcaça e diminuir a agressividade deles. Entre as diferentes técnicas de castração, a cirúrgica é a mais empregada, no entanto produz importante efeito negativo, como dor, edema de bolsa escrotal e exsudação na região da ferida cirúrgica (Crouse et al., 1981; Radostits et al., 2007). A implantação de cânula ruminal, diferentemente da orquiectomia, não é uma cirurgia rotineira, mas com fins experimentais, a qual objetiva facilitar o acesso ao rúmen para estudos de nutrição, fisiologia e metabolismo (Newby et al., 2013). Esses dois procedimentos cirúrgicos, como qualquer outro, promovem dor, sendo necessário avaliar a dimensão desse desconforto, no intuito de diminuir o estresse e proporcionar uma melhor recuperação pós-cirúrgica. Dessa forma, o presente trabalho objetivou comparar o efeito de três AINEs (flunixin meglumine, cetoprofeno e meloxicam) no tratamento da dor pós-operatória de ovinos submetidos à implantação de cânula ruminal e orquiectomia, com base nas alterações comportamentais, hematológicas e bioquímicas.

\section{MATERIAL E MÉTODOS}

O estudo foi aprovado pela Comissão de Ética no Uso de Animais da Faculdade de Medicina Veterinária e Zootecnia da Universidade de São Paulo (Protocolo n ${ }^{\circ}$ 1587/2009).

Foram utilizados 32 ovinos mestiços da raça Santa Inês, machos, com média de $35 \pm 3,5 \mathrm{~kg}$ de peso vivo e 12 meses de idade. Os animais passaram por um período de 30 dias de adaptação às novas condições de manejo, quando foram mantidos em baias e alimentados com dieta basal calculada em $2,7 \%$ do peso vivo, composta de $75 \%$ da matéria seca de feno de capim de coast-cross e $25 \%$ de ração concentrada comercial contendo $14 \%$ de proteína bruta. Foram suplementados com mistura mineral e receberam água ad libitum.

Todos os animais foram submetidos à implantação de cânula ruminal e orquiectomia 
pela técnica aberta (Turner e McIlwraith, 2002) no mesmo dia. Para a realização dos procedimentos cirúrgicos, os animais receberam medicação pré-anestésica com $0,15 \mathrm{mg} / \mathrm{kg}$ de xilazina IV (Xilazin 2\%, Syntec ${ }^{\circledR}$, Brasil) e indução anestésica com $0,01 \mathrm{mg} / \mathrm{kg}$ de diazepan (Diazepam 10mg, Cristália ${ }^{\circledR}$, Brasil) e $4,5 \mathrm{mg} / \mathrm{kg}$ de cetamina IV (Cetamin, Syntec ${ }^{\circledR}$, Brasil). Realizou-se bloqueio em L no flanco esquerdo e infiltração testicular de lidocaína $2 \%(20 \mathrm{~mL}$ por área) sem vaso constritor (Lidovet, Bravet ${ }^{\circledR}$, Brasil).

Os animais foram divididos em três grupos experimentais, tratados no pós-cirúrgico com: GFlu (11 animais - flunixin meglumine $1,1 \mathrm{mg} / \mathrm{kg}, \quad \mathrm{IV}, \quad$ Banamine, Schering-Plough ${ }^{\circledR}$, Canada), GCet (11 animais - cetoprofeno $3,0 \mathrm{mg} / \mathrm{kg}, \mathrm{IV}$, Ketofen $10 \%$, Merial $^{\circledR}$, Canada) e GMel (10 animais - meloxicam - 0,5mg/kg, IV, Maxican 2\%, Ouro Fino $^{\circledR}$, Brasil). Cada indivíduo recebeu três doses de AINE, com intervalos de 24 horas, sendo a primeira dose aproximadamente 10 minutos antes do término das cirurgias. Os animais também receberam duas aplicações de antibioticoterapia com penicilina $G$ procaína, benzilpenicilina $G$ benzatina e di-hidroestreptomicina $(20.000 \mathrm{UI} / \mathrm{kg}$, IM, Penfort PPU, Ouro Fino ${ }^{\circledR}$, Brasil), com intervalos de 72 horas.

Os ovinos foram avaliados em 10 momentos: M0 (pré-avaliação), M1 (10 minutos após a préavaliação), M2 (início da sutura para fixação da cânula ruminal e retirada do primeiro testículo), M3 (logo após as duas intervenções cirúrgicas), M2h (duas horas pós-cirurgia), M12h (12h póscirurgia), M23h (23h pós-cirurgia), M25h (25h pós-cirurgia), M48h (48h pós-cirurgia) e M72h (72h pós-cirurgia). Em todos os momentos, os animais foram submetidos ao exame clínico e à colheita de sangue para avaliação hematológica e bioquímica, até o M48h. Nos M2h, M12h, M23h, M25h e M48h, foi avaliado o comportamento dos animais e atribuído escore de dor adotandose a escala de Lascelles et al. (1994) adaptada para a espécie ovina, a qual atribui valores em uma escala de 0 a 3 para a analgesia da região lesada, nesse caso testículo e fístula ruminal. Atribuiu-se 0 para completa analgesia, sem sinais evidentes de desconforto ou reação à pressão da região lesionada; 1 para boa analgesia, sem sinais evidentes de desconforto, mas com presença de reação à pressão da região lesionada; 2 para analgesia moderada, com alguma evidência de desconforto, acentuando-se quando é feita a pressão da região lesionada; e 3 para a ausência de analgesia, com sinais óbvios de desconforto persistente, intensificando-se mediante pressão da região lesionada.

No exame clínico, avaliou-se frequência cardíaca, frequência respiratória, movimento ruminal e temperatura retal. A avaliação comportamental baseou-se na monitoração individual de cada animal por três minutos, nos tempos predefinidos anteriormente, observandose a presença das seguintes atitudes: andar espontâneo, tremores, bruxismo, interação com outros animais, interesse pelo alimento e adoção de posições antiálgicas (afastamento e distensão dos membros posteriores, arqueamento da região lombar, cabeça curvada para região inguinal ou abdominal).

As amostras de sangue foram coletadas com sistema a vácuo, utilizando-se tubos com anticoagulantes ácido dietileno diamino tetracético (EDTA $\mathrm{K}_{2}$ ), EDTA com fluoreto de sódio e tubos sem anticoagulante, para realização do hemograma, da dosagem de glicose e de outras variáveis bioquímicas, respectivamente.

$\mathrm{O}$ hemograma foi realizado em contador hematológico (ABX Vet, Horiba ${ }^{\circledR}$, França) para determinação do volume globular, número de hemácias, hemoglobina, leucócitos e plaquetas. As dosagens de glicose, proteínas totais, albumina, GGT, AST, CK, creatinina e ureia foram realizadas em analisador bioquímico $\mathrm{Rx}$ Daytona (Randox ${ }^{\circledR}$, Reino Unido), utilizando-se kits comerciais da mesma marca. Todas as análises bioquímicas foram processadas a $37^{\circ} \mathrm{C}$, no entanto os valores da atividade das enzimas AST, GGT e CK apresentados nos resultados estão corrigidos para a temperatura de $30^{\circ} \mathrm{C}$, segundo recomendação de Kaneko et al. (2008).

A concentração de cortisol foi mensurada utilizando-se imunoensaio competitivo por quimioluminescência de fase sólida em aparelho Immulite 1000 (Siemens Healthcare Diagnostics Ltd, Los Angeles, EUA), tendo o ensaio sensibilidade de 5,52mmol/L. A metodologia em questão foi validada para a espécie por Cripps $e t$ al. (2008).

Os dados obtidos durante o período experimental foram analisados quanto a sua distribuição 
normal pela prova de Kolgomorov-Sminorv, e foi avaliada a homogeneidade das variâncias pelo teste de Levene. Os dados que obedeceram à distribuição paramétrica foram submetidos à análise de variância, utilizando-se o procedimento PROC MIXED (SAS), para medidas repetidas no tempo, sendo estudado para cada variável o efeito de tratamento, tempo e interação entre tratamento e tempo. Os dados que não obedeceram à distribuição normal foram submetidos ao teste não paramétrico de KruskalWallis, enquanto os dados comportamentais foram avaliados pelo teste exato de Fisher.

\section{RESULTADOS}

No presente estudo, nos momentos avaliativos iniciais (M0, M1 e M2), os animais dos três grupos experimentais não tinham recebido tratamento analgésico. Esses momentos foram incluídos no estudo para avaliar os animais antes do procedimento cirúrgico e o efeito da anestesia.

A avaliação comportamental mostrou que animais do GCet interagiram menos com outros animais da baia no M25h do que os animais do grupo GFlu e GMel. Nas primeiras duas horas após a cirurgia, a maioria dos animais dos três grupos já andavam espontaneamente e se alimentavam, embora alguns animais apresentassem posições antiálgicas, tremores e bruxismo. Não foi detectada diferença entre grupos para essas variáveis (Tab.1).

Tabela 1. Alterações comportamentais de ovinos submetidos à implantação de cânula ruminal e orquiectomia, tratados com três anti-inflamatórios não esteroidais

\begin{tabular}{|c|c|c|c|c|c|c|}
\hline \multirow[b]{2}{*}{ Variável } & \multirow[b]{2}{*}{ Grupos } & \multirow[b]{2}{*}{$\mathrm{M} 2 \mathrm{~h}$} & \multirow[b]{2}{*}{ M12h } & \multicolumn{3}{|c|}{ Momentos } \\
\hline & & & & M23h & M25h & M48h \\
\hline \multirow{3}{*}{$\begin{array}{l}\text { Interagem com } \\
\text { outros animais }\end{array}$} & GFlu & $\begin{array}{l}90,91 \% \\
(10 / 11)\end{array}$ & $\begin{array}{l}90,91 \% \\
(10 / 11)\end{array}$ & $\begin{array}{c}90,91 \% \\
(10 / 11)^{\mathrm{AB}}\end{array}$ & $\begin{array}{c}81,82 \% \\
(9 / 11)\end{array}$ & $\begin{array}{c}81,81 \% \\
(9 / 11)\end{array}$ \\
\hline & GCet & $\begin{array}{c}45,45 \% \\
(5 / 11)\end{array}$ & $\begin{array}{c}63,64 \% \\
(7 / 11)\end{array}$ & $\begin{array}{l}45,45 \% \\
(5 / 11)^{\mathrm{B}}\end{array}$ & $\begin{array}{c}81,82 \% \\
(9 / 11)\end{array}$ & $\begin{array}{c}72,73 \% \\
(8 / 11)\end{array}$ \\
\hline & GMel & $90 \%(9 / 10)$ & $90 \%(9 / 10)$ & $\begin{array}{c}100 \% \\
(10 / 10)^{\mathrm{A}}\end{array}$ & $90 \%(9 / 10)$ & $\begin{array}{c}100 \% \\
(10 / 10)\end{array}$ \\
\hline \multirow{3}{*}{$\begin{array}{c}\text { Andam } \\
\text { espontaneamente }\end{array}$} & GFlu & $\begin{array}{l}100 \% \\
(11 / 11)\end{array}$ & $\begin{array}{l}100 \% \\
(11 / 11)\end{array}$ & $\begin{array}{c}100 \% \\
(11 / 11)\end{array}$ & $\begin{array}{l}100 \% \\
(11 / 11)\end{array}$ & $\begin{array}{c}100 \% \\
(11 / 11)\end{array}$ \\
\hline & GCet & $\begin{array}{c}72,73 \% \\
(8 / 11)\end{array}$ & $\begin{array}{l}100 \% \\
(11 / 11)\end{array}$ & $\begin{array}{l}100 \% \\
(11 / 11)\end{array}$ & $\begin{array}{l}100 \% \\
(11 / 11)\end{array}$ & $\begin{array}{l}90,91 \% \\
(10 / 11)\end{array}$ \\
\hline & GMel & $\begin{array}{c}100 \% \\
(10 / 10)\end{array}$ & $90 \%(9 / 10)$ & $\begin{array}{l}100 \% \\
(10 / 10)\end{array}$ & $\begin{array}{l}100 \% \\
(10 / 10)\end{array}$ & $\begin{array}{l}100 \% \\
(10 / 10)\end{array}$ \\
\hline \multirow{4}{*}{ Bruxismo } & GFlu & $\begin{array}{c}18,18 \% \\
(2 / 11)\end{array}$ & $0 \%(0 / 11)$ & $0 \%(0 / 11)$ & $0 \%(0 / 11)$ & $0 \%(0 / 11)$ \\
\hline & GCet & $\begin{array}{l}9,09 \% \\
(1 / 11)\end{array}$ & $\begin{array}{c}18,18 \% \\
(2 / 11)\end{array}$ & $\begin{array}{l}9,09 \% \\
(1 / 11)\end{array}$ & $0 \%(0 / 11)$ & $0 \%(0 / 11)$ \\
\hline & GMel & $20 \%(2 / 10)$ & $0 \%(0 / 11)$ & $0 \%(1 / 10)$ & $0 \%(0 / 10)$ & $0 \%(0 / 10)$ \\
\hline & GFlu & $\begin{array}{c}54,55 \% \\
(6 / 11)\end{array}$ & $\begin{array}{l}9,09 \% \\
(1 / 11)\end{array}$ & $\begin{array}{c}18,18 \% \\
(2 / 11)\end{array}$ & $\begin{array}{l}9,09 \% \\
(1 / 11)\end{array}$ & $\begin{array}{c}18,18 \% \\
(2 / 11)\end{array}$ \\
\hline \multirow[t]{2}{*}{ Tremores } & GCet & $\begin{array}{c}63,64 \% \\
(7 / 11)\end{array}$ & $\begin{array}{c}27,27 \% \\
(3 / 11)\end{array}$ & $0 \%(0 / 11)$ & $0 \%(0 / 11)$ & $0 \%(0 / 11)$ \\
\hline & GMel & $\begin{array}{c}60,00 \% \\
(6 / 10)\end{array}$ & $25 \%(1 / 10)$ & $10 \%(1 / 10)$ & $10 \%(1 / 10)$ & $0 \%(0 / 10)$ \\
\hline \multirow{4}{*}{ Alimentam-se } & GFlu & $\begin{array}{l}100 \% \\
(11 / 11)\end{array}$ & $\begin{array}{l}90,91 \% \\
(10 / 11)\end{array}$ & $100 \%(0 / 11)$ & $100 \%(0 / 11)$ & $100 \%(0 / 11)$ \\
\hline & GCet & $\begin{array}{l}90,91 \% \\
(10 / 11)\end{array}$ & $\begin{array}{c}81,82 \% \\
(9 / 11)\end{array}$ & $90 \%(9 / 11)$ & $100 \%(0 / 11)$ & $100 \%(0 / 11)$ \\
\hline & GMel & $90 \%(9 / 10)$ & $90 \%(9 / 10)$ & $100 \%(0 / 10)$ & $90 \%(1 / 9)$ & $100 \%(0 / 10)$ \\
\hline & GFlu & $\begin{array}{c}18,18 \% \\
(2 / 11)\end{array}$ & $\begin{array}{r}9,09 \% \\
(1 / 11)\end{array}$ & $\begin{array}{c}27,27 \% \\
(3 / 11)\end{array}$ & $\begin{array}{c}18,18 \% \\
(2 / 11)\end{array}$ & $\begin{array}{l}9,09 \% \\
(1 / 11)\end{array}$ \\
\hline \multirow[t]{2}{*}{ Posição antiálgica } & GCet & $\begin{array}{l}36,6 \% \\
(4 / 11)\end{array}$ & $\begin{array}{c}27,27 \% \\
(3 / 11)\end{array}$ & $\begin{array}{c}36,36 \% \\
(4 / 11)\end{array}$ & $0 \%(0 / 11)$ & $0 \%(0 / 11)$ \\
\hline & GMel & $10 \%(1 / 10)$ & $30 \%(3 / 10)$ & $10 \%(1 / 10)$ & $0 \%(0 / 10)$ & $0 \%(0 / 10)$ \\
\hline
\end{tabular}

Letras maiúsculas nas colunas significam diferença estatística entre os grupos. 
A avaliação da dor utilizando a escala de Lascelles et al. (1994), adaptada para a espécie ovina, mostrou que o GFlu apresentou maior escore de dor na parede abdominal e na região escrotal no M12h (Tab. 2).

A concentração de cortisol sofreu efeito de tratamento $(\mathrm{P}=0,0354)$ e tempo $(\mathrm{P}<0,0001)$. Maiores concentrações de cortisol foram observadas no M2 para o GMel e nos M3, M12h e M48h para os animais do GFlu. A avaliação entre tempos dentro de cada grupo mostrou comportamento semelhante entre o GFlu e o GCet, em que maiores concentrações de cortisol foram observadas nos M1, M3, M2h e M12h e menores no M72h. Já para o GMel, maiores concentrações de cortisol foram observadas nos M1 e M2 e menores também no M72h (Tab. 3).

Tabela 2. Escore de dor nos testículos e na fístula ruminal obtido com o emprego da escala de análise descritiva descrita por Lascelles et al. (1994), durante o período de avaliação de ovinos submetidos à implantação de cânula ruminal e orquiectomia e tratados com três analgésicos

\begin{tabular}{ccccccc}
\hline \multirow{2}{*}{ Variável } & Grupos & \multicolumn{5}{c}{ Momentos } \\
\cline { 2 - 6 } LAS testículo & GFlu & M2h & M12h & M23h & M25h & M48h \\
& GCet & $2(0-2)$ & $2(1-2)^{\mathrm{A}}$ & $1(0-2)$ & $1(0-2)$ & $1(0-2)$ \\
& GMel & $1(0-2)$ & $1(0-3)^{\mathrm{AB}}$ & $1(0-3)$ & $1(0-2)$ & $1(1-2)$ \\
& GFlu & $1(0-2)$ & $1(0-2)^{\mathrm{B}}$ & $1(1-2)$ & $1(1-2)$ & $1(1-2)$ \\
\multirow{3}{*}{ LAS rúmen } & GCet & $2(0-2)$ & $1(0-2)^{\mathrm{A}}$ & $1(1-2)$ & $1(0-2)$ & $1(0-2)$ \\
& GMel & $1(0-2)$ & $1(0-2)^{\mathrm{B}}$ & $1(0-3)$ & $1(0-2)$ & $1(1-2)$ \\
& & &
\end{tabular}

Letras maiúsculas nas colunas significam diferença estatística entre os grupos.

Tabela 3. Média e desvio-padrão das concentrações de cortisol (mmol/L) e glicose (mmol/dL) e das frequências cardíaca (bat/min) e respiratória $(\mathrm{mov} / \mathrm{min})$ de ovinos submetidos à implantação de cânula ruminal e orquiectomia, os quais receberam três protocolos analgésicos

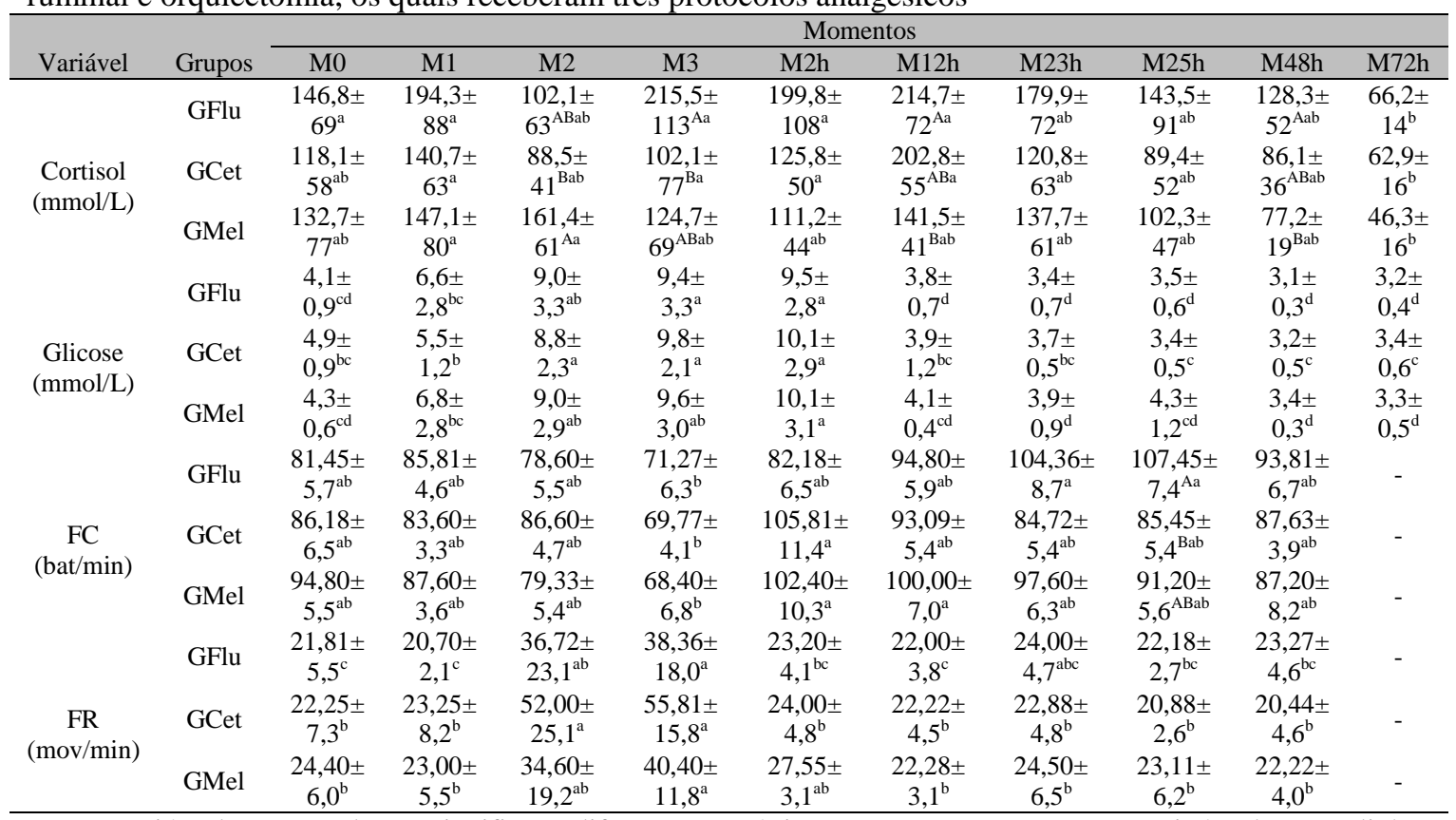

Letras maiúsculas nas colunas significam diferença estatística entre os grupos. Letras minúsculas nas linhas significam diferença entre os momentos dentro do grupo.

Para a concentração de glicose, foi observado apenas efeito de tempo $(\mathrm{P}<0,0001)$, em que maiores valores de glicose foram observados a partir do M2 até o M2h para todos os grupos, ocorrendo redução nos tempos posteriores (Tab.
3). Não foi observado efeito de tratamento para a concentração de proteína total e albumina, apenas efeito de tempo $(\mathrm{P}<0,0001)$. Ocorreu redução na concentração de proteína total no M3 para todos os grupos (dados não mostrados). A 
avaliação da função renal demonstrou que tanto a concentração de ureia quanto a de creatinina não foram afetadas pelos tratamentos. $\mathrm{Na}$ avaliação entre tempos, constatou-se que, no M72h, as concentrações de ureia diminuíram nos três grupos (Fig. 1).

A atividade de AST e CK não foi alterada pelos tratamentos, ocorreu apenas efeito de tempo. Os três grupos apresentaram maiores valores de AST a partir do M12h, os quais permaneceram elevados nos momentos posteriores, enquanto maiores valores de $\mathrm{CK}$ foram observados no M12h, ocorrendo redução nos tempos posteriores (Fig. 1). Não foram observadas alterações para a atividade da GGT.

Os tratamentos não alteraram a frequência cardíaca, a frequência respiratória e a temperatura corporal. Apenas no momento M25h, os animais do GFlu apresentaram maior frequência cardíaca. Na comparação entre tempos, observou-se que a frequência cardíaca diminuiu no M3 (Tab. 3), enquanto a temperatura corporal diminuiu do M2 ao M2h para os três grupos. Maiores valores da frequência respiratória foram observados do M2 ao M3 para todos os grupos, ocorrendo redução nos momentos posteriores. A avaliação ruminal mostrou que os animais tiveram redução da motilidade ruminal no $\mathrm{M} 2$ e no $\mathrm{M} 3$, enquanto no $\mathrm{M} 2 \mathrm{~h}$ os animais do GMel tiveram menor motilidade quando comparados aos animais dos outros grupos (Fig. 1).

A avaliação hematológica demonstrou que não houve efeito de tratamento para o número de neutrófilos, o volume globular, o número de hemácias, a concentração de hemoglobina e plaquetas. $\mathrm{O}$ volume globular e o número de hemácias e hemoglobina reduziram-se significativamente do M2 ao M3 nos três grupos (Fig. 1). Animais GCet e GMel tiveram redução no número de leucócitos no M3 e de hemácias do M2 ao M2h (Fig. 1).

\section{DISCUSSÃO}

A mensuração da dor em animais tem sido um grande desafio, já que esses, diferentemente dos humanos, não fazem uso da linguagem verbal, assim índices fisiológicos, bioquímicos e comportamentais são utilizados para fazer julgamento sobre o nível de dor induzido por procedimentos invasivos, como os que foram desenvolvimentos por este estudo (Thuer et al., 2007).

A concentração de cortisol tem sido utilizada em diversos estudos como marcador indireto de estresse e dor em ruminantes. Neste estudo, foi observada variação na concentração desse hormônio em momentos específicos, indicando que os animais que receberam flunixin meglumine apresentaram maiores concentrações de cortisol, enquanto os que receberam meloxicam tiveram menores concentrações desse hormônio. No entanto, durante o período cirúrgico (M2) e no pós-cirúrgico imediato (M3), ocorreu aumento do cortisol no GMel e no GFlu, respectivamente (Tab. 3). Essa elevação pode estar associada à resposta individual do grupo diante do estresse da manipulação, já que a anestesia foi semelhante para os três grupos. Nesse contexto, sabe-se que as concentrações de cortisol podem se elevar de 15 a 20 minutos após o estímulo, como pode ter ocorrido devido ao início da incisão do testículo e à sutura da cânula no M2, ou devido ao estresse de manipulação no pós-operatório imediato no M3 (Luger et al., 1987; Raff et al., 1987), momento em que os animais eram colocados em estação e estimulados a caminhar até a baia.

Mellema et al. (2006) observaram que, mesmo utilizando anestesia local, essa não reduziu a concentração de cortisol nem o comportamento de dor durante os primeiros 120 minutos após a castração com burdizzo em ovinos. Esse fato foi associado à lesão de vias aferentes do plexo pampiniforme, que levariam à dor visceral, ocorrendo o mesmo fato quando da castração cirúrgica (Cottrell e Molony, 1995). No entanto, é importante salientar que esses autores não utilizaram AINEs no pós-cirúrgico, o que possivelmente promoveu aumento do cortisol por um período prolongado após a castração. 


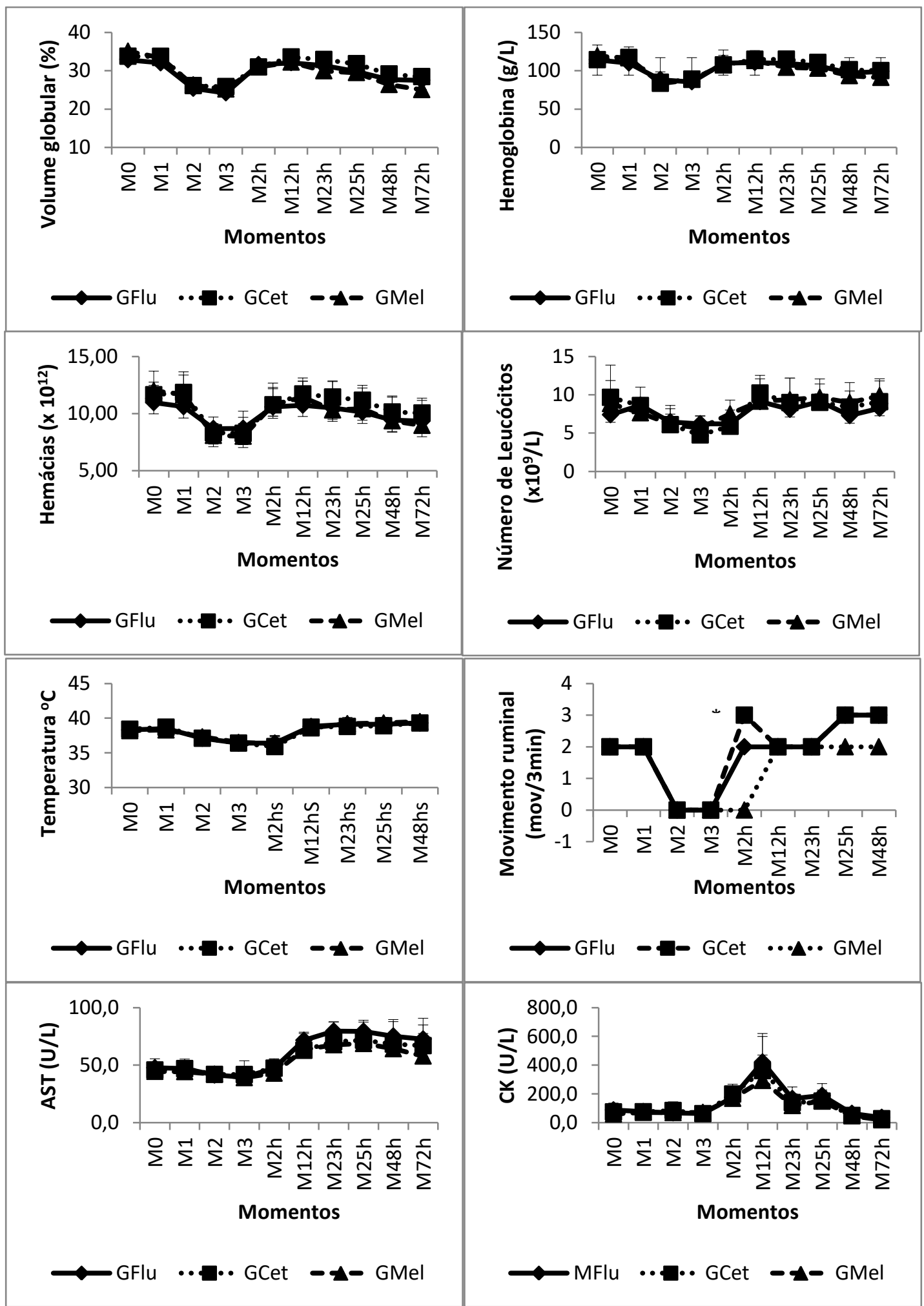

Figura 1. Valores médios das variáveis hematológicas, clínicas e bioquímicas dos ovinos submetidos à implantação de cânula ruminal e orquiectomia, tratados com três protocolos analgésicos. *Significa diferença entre os grupos experimentais no momento estudado. 
Segundo Anderson e Muir (2005), o flunixin é um medicamento que promove excelente analgesia visceral, porém menor em lesões musculoesqueléticas. Como no presente estudo o estímulo doloroso incluía procedimentos de dor visceral e musculoesquelética, possivelmente o flunixin não tenha sido tão eficiente em combater a dor quanto o meloxicam, fato que proporcionou aumento do cortisol nos momentos M12h e M48h. Newby et al. (2013), ao compararem o cetoprofeno e o meloxicam na analgesia de bovinos após a implantação de cânula ruminal, concluíram que esse procedimento causa desconforto, aumenta a frequência cardíaca e respiratória quando comparado ao momento basal. No entanto, esses autores não observaram diferença entre esses dois AINES no tratamento da dor pós-cirúrgica. No M12h e no M48h, as concentrações de cortisol do GCet não diferiram do GFlu e GMel.

Além das determinações da concentração de cortisol, a avaliação da dor também tem se baseado na observação do comportamento individual desses animais (Molony e Kent, 1997; Thuer et al., 2007). No presente estudo, utilizouse a escala de Lascelles et al. (1994), a qual, embora tenha sido desenvolvida para mensurar a dor em felinos, demonstrou ser eficiente para pequenos ruminantes, já que os animais que receberam flunixin meglumine apresentaram maior escore de dor tanto na ferida abdominal como na região escrotal (Tab. 2) quando comparados aos animais dos demais grupos no $\mathrm{M} 12 \mathrm{~h}$, fato esse corroborado pela maior concentração de cortisol nesse mesmo grupo no momento avaliado.

Embora alguns autores relacionem a redução do apetite com o estado de saúde, ou mesmo como um sinal de dor ou desconforto (Radostits et al., 2007; Newby et al., 2013), neste estudo foi observado no M2h que os animais já apresentavam interesse pelo alimento (Tab. 1), indicando que esses, mesmo após o estímulo doloroso, buscam alimento. Porém, não foi avaliado o consumo individual ou a matéria seca por animal, como foi realizado por Newby et al. (2013), que observaram que animais submetidos à implantação de cânula ruminal diminuíram o consumo de matéria seca no primeiro dia de cirurgia.
A ausência de interação entre os animais foi a única variável comportamental que apresentou diferença na comparação entre os grupos. O GCet, no M23h, teve maior número de ovinos que não interagiam com outros animais, ou seja, permaneciam parados e isolados em um canto da baia, comportamento esse incomum para pequenos ruminantes, quando em convívio coletivo. Embora a adoção de posição antiálgica não diferisse entre os grupos, no M23h dos cinco animais que não interagiam com os demais, quatro apresentaram posição antiálgica, indicando que os animais possivelmente estavam sentindo dor (Tab. 1). O cetoprofeno possui meia-vida de duas horas quando administrado por via intramuscular e $80 \%$ da dose é eliminada na urina com 24 horas da administração (Merial, 2002 - MERIAL-CANADA. Anafen injection (large animal) in Compendium of Veterinary Products. CD ed. A. Arrioja-Dechert, ed. North American Compendiums Inc., yPort Huron, MI, 2002). Como a avaliação foi realizada 23 horas após a última aplicação de cetoprofeno, possivelmente isso possibilitou a detecção desse comportamento anormal nos animais.

Maiores concentrações de glicose foram observadas nos três grupos, do M2 ao M2h. Essa alteração pode estar ligada ao efeito hiperglicemiante da xilazina, droga que possui efeito sedativo e analgésico de 30 a 40 minutos (Abrahamsen, 2008), porém estudo em bovinos demonstrou que o efeito hiperglicemiante pode perdurar por 3,5 a cinco horas após a administração desse fármaco (Kasuya et al., 1996). O efeito hiperglicemiante desse $\alpha_{2}$ agonista se deve à ação direta sobre o fígado, estimulando a glicogênese e promovendo menor utilização da glicose pelas células do corpo devido à diminuição das concentrações de insulina no sangue (Raptopoulos e Weaver, 1984; Thurmon et al., 1982).

Os animais dos três grupos apresentaram redução do volume globular e do número de hemácias e hemoglobina do M2 ao M3, que ficaram abaixo dos valores de referência para ovinos (volume globular - 27-45\%; número de hemácias - 9-15 x $10^{12} \mu \mathrm{L}$ e hemoglobina - 9-15g/dL) (Weiss e Wardrop, 2010). Essa redução nos valores hematológicos pode estar associada ao efeito da xilazina na diminuição do tônus simpático, levando ao sequestro das células vermelhas no baço. Kullmann et al. (2014) observaram que 
equinos tratados com $\alpha_{2}$-agonista apresentaram redução do volume globular e do número de hemácias, sendo esse evento acompanhado de aumento da espessura do baço. A concentração de proteína total também se reduziu no M3 para os três grupos, concomitantemente à redução do volume globular, porém os valores ficaram dentro do intervalo de referência $(6,0-7,9 \mathrm{~g} / \mathrm{dL})$ (Radostistis et al., 2007), enquanto a concentração de albumina não foi afetada.

O número de leucócitos permaneceu dentro dos valores de referência, o que diverge dos achados de Fatoretto (2009), que, ao comparar a técnica de castração aberta e fechada, observou aumento significativo do número de leucócitos 24 e 48 horas após o procedimento cirúrgico, o que indica que, no presente estudo, o uso dos AINEs foi eficiente em controlar o processo inflamatório decorrente dos procedimentos cirúrgicos.

O número de plaquetas não sofreu nenhuma alteração, mesmo os animais tendo passado por um processo de perda sanguínea e lesão tecidual. É importante salientar que a não alteração dessa variável indica que não houve efeito adverso ao uso dos AINES, já que a COX-1 é uma enzima essencial para a atividade das plaquetas.

Frequências cardíaca e respiratória são marcadores indiretos de dor em ruminantes. Apesar disso, nem sempre a frequência cardíaca é um indicador de dor fidedigno, como se pôde perceber nos momentos em que ocorreu aumento de cortisol, pois este nem sempre foi acompanhado de aumento da frequência cardíaca. No presente estudo, quando a frequência cardíaca foi comparada dentro de cada grupo, observou-se que, nos três grupos, houve diminuição da frequência cardíaca no M3, causada possivelmente pelo efeito da xilazina, que promove bradicardia e redução do débito cardíaco, devido à ativação dos $\alpha_{2}$ adrenoceptores pré-sinápticos, que inibem a exocitose da noradrenalina (De Jonge et al., 1981; Yamashita et al., 2000).

Uma das implicações da xilazina é a bradipneia, no entanto, no M2 e no M3, foi observado que os grupos experimentais apresentaram aumento da frequência respiratória, o que deve estar ligado ao efeito compensatório da bradipneia provocado pela xilaziana, em que ocorre aumento da $\mathrm{pCO}_{2}$, levando à ativação de barorreceptores, que é rapidamente compensada pelo aumento da frequência respiratória (Kastner, 2006; Plummer e Schleining, 2013). Outro efeito observado pelo uso da xilazina é a diminuição da motilidade ruminal, como pode ser observado no M2 e no M3 para os três grupos. Tal fato está associado à inibição da contração primária e secundária do rúmen mediada por esse fármaco (Ribeiro et al., 2012).

A temperatura corporal também pode ser afetada pela xilazina, que tem ação inibitória sobre o centro termorregulador do hipotálamo, devido à redução na liberação de noradrenalina em terminais pré-sinápticos (Suprenant e North, 1988). Neste estudo, foi observado que os três grupos tiveram redução significativa dessa variável do M2 ao $\mathrm{M} 2 \mathrm{~h}$, sendo este último o momento em que foi observado maior número de animais com tremores.

Sabe-se que a utilização de AINEs pode afetar a função gastrointestinal, plaquetária e renal, sendo importante também avaliar o fígado, órgão responsável pela metabolização desses fármacos. A concentração de ureia diminuiu no M72h, mas, durante todo o período experimental, os valores ficaram dentro do intervalo de referência para a espécie, assim como a concentração de creatinina, indicando que a utilização desses AINEs por três dias não afetou a função desse órgão. A concentração de AST aumentou após o M12h, mas não ultrapassou os valores de referência (60-280U/L). No entanto, esse incremento possivelmente está ligado ao tempo de decúbito em que os animais ficaram para a realização do procedimento cirúrgico, o qual pode ter provocado microlesões musculares, o que é corroborado pelo aumento da $\mathrm{CK}$ nesse mesmo período (Kaneko et al., 2008). Com base nos resultados obtidos, pode-se concluir que a ação analgésica do meloxicam foi maior em animais submetidos à implantação de cânula ruminal e à orquiectomia quando comparado ao flunixin meglumine e ao cetoprofeno. 


\section{REFERÊNCIAS}

ABRAHAMSEN, E.J. Chemical restraint in ruminants. Vet. Clin. Food Anim., v.24, p.227-243, 2008.

$\begin{aligned} & \text { ADEGBOYEGA, P.A.; } \begin{array}{c}\text { OLOLADE, } \\ \text { Immunohistochemical O. }\end{array} \\ & \text { cyclooxygenase-2 in normal kidneys. App. } \\ & \text { Immunol. } \text { Mol. } \text { Morphol., v.12, p.71-74, 2004. }\end{aligned}$

ANDERSON, D.E.; MUIR, W.W. Pain management in ruminants. Vet. Clin. Food Anim., v.21, p.19-31, 2005.

COETZEE, J.F.; KUKANICH, B.; MOSHER, R. et al. Pharmacokinetics of intravenous and oral meloxicam in ruminant calves. Vet. Ther. Res. App. Vet. Med., v.10, p.1-8, 2009.

COTTRELL, D.F.; MOLONY, V. Afferent activity in the superior spermatic nerve of lambs: the effects of application of rubber castration rings. Vet. Res. Commun., v.19, p.503-515, 1995.

CRIPPS, L.R.; GREEN, L.R.; THOMPSON, J. The effect of maternal body condition score before and during pregnancy on the glucose tolerance of adult sheep offspring. Reprod. Sci., v.15, p.448-456, 2008 .

CROUSE, J.D., BUSBOOM, J.R.; FIELD, R.A. et al. The effects of breed, diet, sex, location and slaughter weight on lamb growth, carcass composition and meat flavor. J. Anim. Sci., v.53, p.376-386, 1981

DE JONGE, A.; TIMMERMANS, P.B.; VAN ZWEITEN, P.A. Participation of cardiac presynaptic $\alpha 2$-adrenoceptors in the bradycardic effects of clonidine and analogues. NaunynSchmiedeberg's Arch. Pharmacol., v.137, p.8-12, 1981.

FATORRETO, B. Perfil inflamatório e cicatricial em ovinos submetidos à orquiectomia. An. Prod. Inic. Cient. Disc., v.12, p.43-55, 2009.

FITZPATRICK, J.; SCOTT, M.; NOLAN, M. Assessment of pain and welfare in sheep. Small Ruminant Res., v.62, p.55-61, 2006.

KANEKO, J.J.; HARVEY, J.W.; BRUSS, M.L. Clinical biochemistry of domestic animals. 6.ed. San Diego: Elsevier, 2008. [918p.].

KÄSTNER, S.B.R. A ${ }^{2}$-agonistas in sheep: a review. Vet. Anaest. Anal., v.33, p.79-96, 2006.
KASUYA, E.; HODATE, K.; MATSUMOTO, M. et al. The effects of xylazine on plasma concentrations of growth hormone, insulin-like growth factor-1, glucose and insulin in calves. Endoc. J., v.43, p.145-149, 1996.

KULLMANN, A.; SANZ, M.; FOSGATE, G.T. et al. Effects of xylazine, romifidine, or detomidine on hematology, biochemistry, and splenic thickness in healthy horses. Can. Vet. J., v.55, p.334-340, 2014.

LASCELLES, B.D.X.; BUTTERWORTH, S.J.; WATERMAN, A.E. Postoperative analgesic and sedative effects of carprofen and pethidine in dogs. Vet. Rec., v.134, p.187-191, 1994.

LEES, P.; LANDONI, M.F.; GIRAUDEL, J. et al. Pharmacodynamics and pharmacokinetics of nonsteroidal anti-inflammatory drugs in species of veterinary interest. J. Vet. Pharmacol. Ther., v.27, p.479-490, 2004.

LORENA, S.E.R.S.; LUNA, S.P.L.; LASCELLES, B.D.X. et al. Atitude of Brazilian veterinarians in the recognition and treatment of pain in horses and cattle. Vet. Anaesth. Analg., v.40, p.410-418, 2013.

LUGER, A.; DEUSTER, P.A.; KYLE, S.B. et al. Acute hypothalamic-pituitary-adrenal responses to stress of treadmill exercise: physiologic adaptations to physical training. N. Engl. J. Med., v.316, p.1309-1315, 1987.

MELLEMA, S.C.; DOHERR, M.G.; WECHSLER, B. et al. Influence of local anaesthesia on pain and distress induced by two bloodless castration methods in young lambs. Vet. J., v.172, p.274-283, 2006.

MOLONY, V.; KENT, J.E. Assessment of acute pain in farm animals using behavioral and physiological measurements. J. Anim. Sci., v.75, p.266-272, 1997.

MUIR, W.W.; WOOLF, C.J. Mechanisms of pain and their therapeutic implications. J. Am. Vet. Med. Assoc., v.219, p.1346-1356, 2001.

NEWBY, N.C.; TUCKER, C.B.; PEARL, D.L. et al. A comparison of 2 nonsteroidal antiinflammatory drugs following the first stage of a 2stage fistulation surgery in dry dairy cows. J. Dairy Sci., v.96, p.6514-6519, 2013.

PLUMMER, P.J.; SCHLEINING, J.A. Assessment and management of pain in small ruminants and camelids. Vet. Clin. Food Anim., v.29, p.185-208, 2013. 
RADOSTITS, O.M.; GAY, C.C.; HINCHCLIFF, K.W.; CONSTABLE, P.D. Veterinary medicine: a textbook of the diseases of cattle, sheep, goats, pigs and horses. 10.ed. Philadelphia: Saunders Elsevier, 2007. 2158p.

RAFF, H.; NORTON, A.J.; FLEMMA, R.J. et al. Inhibition of the adrenocorticotropin response to sugery in humans: interaction between dexamethasone and fentanyl. J. Clin. Endocrinol. Metab., v.65, p.295-298, 1987.

RAPTOPOULOS, D.E.; WEAVER, B.M.Q. Observations following intravenous xylazine administration in steers, Vet. Rec., v.114, p.567$569,1984$.

RIBEIRO, G.; DÓRIA, R.G.S.; NUNES, A.L. et al. Efeitos de detomidina e xilazina intravenosa sobre as variáveis basais e respostas comportamentais em bovinos. Arq. Bras. Med. Vet. Zootec., v.64, p.1411-1417, 2012.

SILVA, S.T.G.; TENÓRIO, A.P.M.; AFONSO, J.A.B. et al. Fisiopatologia da dor em ruminantes e equinos. Med. Vet., v.5, p.18-23, 2011

SUPRENANT, A.; NORTH, R.A. Mechanism of synaptic inhibition by noradrenaline acting at alpha 2-adrenoceptors. Proc. R. Soc. Lond., v.234, p.85114,1988
THUER, S.; MELLEMA, S.; DOHERR, M.G. et al. Effect of local anaesthesia on short- and longterm pain induced by two bloodless castration methods in calves. Vet. J., v.173, p.333-342, 2007.

THURMON, J.C.; NEFF-DAVIS, C.; DAVIS, L.E. et al. Xilazine hydrochloride-induced hyperglycemia and hypoinsulinemia in thoroughbred horses. J. Vet. Pharmacol. Ther., v.5, p.241-245, 1982.

TURNER, A.S.; McILWRAITH, C.W. Técnicas cirúrgicas em animais de grande porte. São Paulo: Roca, 2002. 341p.

WEISS, D.J.; WARDROP, J. Schalm's veterinary hematology. 6.ed. Iowa: Willey Blackwell, 2010. p.837.

WHAY, H.R.; HUXLEY, J.N. Pain relief in cattle: a practitioner's perspective. Cattle Pract., v.13, p.81-85, 2005.

WHAY, H.R.; WEBSTER, A.J.F.; WATERMANPEARSON, A.E. Role of ketoprofen in the modulation of hyperalgesia associated with lameness in dairy cattle. Vet. Rec., v.3, 2005.

YAMASHITA, K.; TSUBAKISHITA, S.; FUTAOKA, S. et al. Cardiovascular effects of medetomidine, detomidine and xylazine in horses. J. Vet. Med. Sci., v.62, p.1025-1032, 2000 